\title{
Epidemic of Non-Communicable Diseases and Its Control
}

\section{Bhattarai MD}

General Medicine, Diabetes and Endocrinology Unit

Bir Hospital, National Academy of Medical Sciences, Kathmandu

Non-communicable Diseases (NCD) principally includes four common diseases, viz. diabetes, cardiovascular diseases (CVD), cancers and chronic respiratory diseases, which caused $63 \%$ of global deaths in $2008 .{ }^{1}$ CVD alone accounts for nearly $30 \%$ of all deaths worldwide and $27 \%$ in low-income and middle-income countries. About 366 million people were reported to have diabetes in 2011 worldwide. ${ }^{2}$ In low- and middle-income countries, NCD is not only common but also kills people at younger age. ${ }^{1}$ Nepal is also facing the surging burden of NCD. ${ }^{3}$ In a study across Nepal, $36.5 \%$ of admitted patients suffered from NCD out of which $38 \%$ were having heart diseases followed by chronic obstructive pulmonary diseases (33\%) and diabetes and cancer $(29 \%){ }^{3}$ In a nation-wide population study of urban Nepal, the prevalence of diabetes was $14.6 \%$ and $19 \%$ among the people 20 years and above and 40 years and above respectively with accompanying hypertension in $22.7 \%$ in the later group. ${ }^{4}$

The major advantage of considering the four prevalent diseases as NCD is for planning their control cohesively due to their common risk-factors like physical inactivity, unhealthy diet, tobacco use, alcohol use, hypertension, poor mother and child health care and others. Physical inactivity is estimated to be a causative factor in 10 to 16 percent each of breast, colon and rectal cancers and diabetes, and in about 22\% of CVD. ${ }^{5}$ Unhealthy diet, especially inadequate fruit and vegetable, excess salt, sugar and fat and consumption of trans-fatty acids (TFA) increases the risk of various NCD. ${ }^{1}$ TFA decreases HDL-cholesterol and increase LDL cholesterol with increased risk of CVD, insulin resistance, and risk for diabetes. Inadequate water intake is another unhealthy lifestyle. ${ }^{6}$ People not taking adequate amount of water may replace it with other energyyielding beverage and alcohol. More than $20 \%$ of total daily energy intake and more than $80 \%$ of total water intake may be derived from beverages. ${ }^{7}$ Active participation in Healthy Cooking Societies and Healthy Physical Activity Societies can bridge the gap between the available scientific evidences and actual practice. ${ }^{6}$ Risks of CVD, diabetes and certain cancers increase steadily with increasing body mass index (BMI). ${ }^{1}$ The normal ranges of BMI are 18.5-24.9 kg/m2 for Europids (early1990s), 18.5-22.9 kg/m2 for Asians (2000), and $18.0-22.9 \mathrm{~kg} / \mathrm{m} 2$ for Asian Indians (2009). ${ }^{8,9}$ Tobacco use and indoor and outdoor air pollution are wellknown causes of various cancers, chronic respiratory diseases and CVD. 5

Alcohol consumption is one of top ten leading risk-factors of avoidable and attributable disease burdens worldwide. ${ }^{5}$ More than half of these deaths occur from NCD including cancers and CVD. ${ }^{1}$ Breast cancer risk is higher for women consuming moderate to high levels of alcohol compared with abstainers, and there is a significant dose response relationship beginning with intakes as low as one to two drinks per day. Several types of gastrointestinal cancer are linked to alcohol consumption even at low levels of intake. ${ }^{5}$ Confusion is created by some benefits of alcohol observed with CVD in observational studies. However the main difficulty of observational studies that compare abstainers with alcohol users is that alcohol use is not distributed randomly among individuals; people who abstain are different than those who drink in more ways than just how much alcohol they consume. ${ }^{10}$ There is no randomized controlled trial required for evidence-based practice showing benefits with alcohol consumption if any. A longitudinal cohort study found no evidence of CVD benefit from moderate alcohol consumption among those with other healthy lifestyle traits like good diet, good exercise and no smoking. ${ }^{11}$ Therefore, such healthy lifestyle should be recommended not alcohol. It is even prudent to propose toast on official occasions, at least by medical professionals by drinking glass of water instead of alcohol highlighting both hazards of alcohol and benefits of water. ${ }^{6}$

Hypertension causes more deaths in the world than any of the above four main behavioral risk factors. ${ }^{1}$ Ultimately, most people with hypertension require more than one medicine to control their blood pressure. Combination of appropriate anti-hypertensive drugs in one tablet appears useful for patient compliance. ${ }^{12}$ This is in contrast to diabetes where combination of the safe optimal first-line drug metformin with other drugs like sulphonylureas is associated with risk of hypoglycemia and needs to be avoided. Availability of such irrational combinations will promote unnecessary, early and longer use of the combined drugs with 
reduced dose of metformin due to difficulty in dose adjustment.

About one fifth of global cancer burden is attributable to some specific chronic infections. ${ }^{1}$ Sexual and/or blood-borne transmission of hepatitis B virus (HBV), hepatitis C virus, human papilloma virus (HPV) and human immunodeficiency virus (HIV) is related to the occurrence of a number of cancers like hepatic, cervical, and others. In Nepal, the burden of cancers is reported to be higher in females. ${ }^{3}$ The $100 \%$ condom program in Thailand was quite successful in preventing HIV transmission. ${ }^{13}$ The educational message like "Avoid multiple sex partners" is inappropriate in the developing countries where extra-marital sex is most likely to involve commercial sex workers with poor health and economic conditions. The message for safer sex needs to be relevant and acceptable to the local situation like "Avoid any sex outside marriage or else use condom consistently". ${ }^{14}$ Apart from 'The 100\% Safer Sex Program', Universal precautions in the health settings and appropriate immunization against HBV and HPV are other considerations. ${ }^{6} \mathrm{HBV}$ vaccination to infants is recently introduced in the immunization program. Vaccination should be provided to health professionals and trainee-students against diseases to which they are exposed during the course of their duty as an urgent priority to protect them and their spouses and offspring. In a study in Nepal, nurses and non-professional health-staff were significantly more affected by HBV infection than others. ${ }^{15}$

In some limited and nation-wide surveillance studies conducted in Nepal, prevalence of both behavioral and intermediate risk factors for major NCDs like obesity and hypertension were remarkably high. ${ }^{16,17}$ Tobacco products causing NCD also include smokeless-tobacco like snuff, gutkha, gul, chimo, mawa, nass, pan masala, tambaku and others. ${ }^{18}$ These are commonly consumed in our regions. Similarly, partially hydrogenated fats, vegetable ghee, a clarified butter commonly used in cooking in South-East Asian countries contains trans-fatty acids (TFA) levels as high as $50 \% .{ }^{19}$ Industrialized countries aim to reduce the consumption of industrial TFA to less than $1 \%$.

Above behavioral risk-factors alone, however, cannot explain the rapid rise of diabetes and CVD. The intricate relations of NCD with Mother and Child Health (MCH) have assumed prime importance for NCD control. ${ }^{6,20}$ The dissociation in metabolic states of fetal life with nutritional want and adult life with nutritional surfeit causes rapid transition in the nutritional status of population which exposes the fetus to an adverse environment in utero leading to permanent programming of tissue function and a risk of cardiovascular and renal diseases, obesity and metabolic syndrome. ${ }^{21}$ The nutritional supplementation program to infants has been implemented in Nepal. Similar programs for females of reproductive age in rural and poorer sectors of society will help to improve the nutritional and metabolic status of fetus during pregnancy. The other important factor in relation to NCD and $\mathrm{MCH}$ is the metabolic imprinting of fetus due to clinical or subclinical maternal hyperglycemia during pregnancy with subsequent substantially enhanced risk of diabetes, obesity, CVD and other NCDs in the offspring. ${ }^{20}$ Risk of glucose intolerance even in non-pregnant adult appears to increase when BMI rises more than the middle of the recommended number. ${ }^{5}$ The pre-pregnancy weight of women should be optimum, preferably below the middle of the recommended $\mathrm{BMI}$ for the population, to decrease the risk of even sub-clinical hyperglycemia during pregnancy leading to fetal metabolic programming. Lastly in relation to $\mathrm{MCH}$, breastfeeding needs to be promoted vigorously as it reduces the risks of obesity, cancer, CVD and diabetes not only in the offspring but also in mothers. ${ }^{22}$ Thus for the adequate and timely control of diabetes and other NCD epidemic, Mother and Child Health has become the key priority. This can be effectively summarized and highlighted by poster campaign. ${ }^{23}$

As NCDs are multi factorial, their prevention and control should be attempted by regulating those factors. The following important ten measures are recommended to reduce the burden NCD. 1) regular physical activity 2) healthy diet 3) healthy body weight 4) avoiding tobacco use in any form and reducing indoor and outdoor air pollution 5) avoiding alcohol 6) universal safer sex, universal precautions and vaccination against HBV and HPV 7) control of hypertension 8) nutritional supplementation to females of reproductive age in rural and poorer sectors of society 9) breastfeeding; and 10) maintenance of optimum pre-pregnancy weight of women, preferably below the middle of the recommended BMI range.

\section{REFERENCES}

1. WHO. Global Status Report on Noncommunicable Diseases 2010. Geneva: WHO, 2011.

2. International Diabetes Federation. IDF Diabetes Atlas. 5th Ed. Brussels: International Diabetes Federation, 2011.

3. Nepal Health Research Council. Prevalence of Non Communicable Disease in Nepal: Hospital Based Study. Kathmandu: Nepal Health Research Council, 2010.

4. Shrestha UK, Singh DL, Bhattarai MD. The prevalence of hypertension and diabetes defined by fasting and 2-h plasma glucose criteria in urban Nepal. Diabetic Medicine 2006; 23: 1130-1135.

5. WHO. World health report 2002: Reducing risks, promoting healthy life. Geneva: WHO, 2003.

6. Bhattarai MD. Primary Prevention and Control of Type 2 Diabetes and Other Non-Communicable Diseases by Healthy Lifestyle Program. Kathmandu: General Welfare Pratisthan, 2010. 
7. McKiernan, F., Hollis J.H., McCabe, GP., Mattes, R.D. Thirst-drinking, hunger-eating; tight coupling? J Am Diet Assoc. 2009; 109: 486.

8. World Health Organization, International Association for the Study of Obesity, International Obesity Task Force. The Asia-Pacific perspective: Redefining obesity and its treatment. Sydney: Health Communications, 2000

9. Misra, A., Chowbey, P., Makkar, B.M., Vikram, N.K., Wasir, J.S., Chadha, D., et al for Consensus Group. Consensus statement for diagnosis of obesity, abdominal obesity and the metabolic syndrome for Asian Indians and recommendations for physical activity, medical and surgical management. J Assoc Physicians India 2009; 57: 163.

10. Kenneth J, Mukamal KJ, Fletcher RH, Sokol HN.Overview of the risks and benefits of alcohol consumption, UpToDate 2010; 18: 1.

11. Britton A, Marmot MG, Shipley M. Who benefits most from the cardioprotective properties of alcohol consumption-health freaks or couch potatoes? J Epidemiol Community Health 2008; 62: 905-8.

12. Bhattarai MD. Combination anti-hypertensives in WHO essential medicine list. Lancet 2005; 366: 633-634

13. UNAIDS. UNAIDS and WHO: The HIV/AIDS situation in mid-1996 Global and regional highlights. Geneva: UNAIDS, 1996.

14. Bhattarai MD. Wait AIDS is Your Own Problem as well. Kathmandu: General Welfare Pratisthan 1997.

15. Shrestha SK, Bhattarai MD. Study of hepatitis B among different categories of health care workers. J Coll Physicians Surg Pak 2006; 6 : 108-111.
16. Ministry of Health and Population, Government of Nepal. Surveillance of Risk Factors for Non-Communicable Diseases in Nepal: Report from Kathmandu Metropolitan City. Kathmandu: Ministry of Health and Population, 2010.

17. Ministry of Health and Population, Government of Nepal, Society for Local Integrated Development Nepal (SOLID Nepal) and WHO. WHO STEPS Surveillance: Non-Communicable Diseases Risk Factors Survey. Kathmandu: Ministry of Health and Population, Government of Nepal, Society for Local Integrated Development Nepal (SOLID Nepal) and WHO, 2008.

18. A policy statement from the American Heart Association. Impact of smokeless tobacco products on cardiovascular disease: Implications for policy, prevention, and treatment. Circulation 2010; 122: 1-25.

19. Chan JCN, Malik V, Jia W, Kadowaki T, Yajnik CS, Yoon KH, et al. Diabetes in Asia: Epidemiology, risk factors, and pathophysiology. JAMA 2009; 301: 2129.

20. Bhattarai, M.D. Three Patterns of Rising type 2 Diabetes Prevalence in the World: Need to Widen the Concept of Prevention in Individuals into Control in the Community. J Nep Med Assoc. 2009; 48: 173-9.

21. Hales CN, Barker DJP. Type 2 (non-insulin-dependent) diabetes mellitus: the thrifty phenotype hypothesis. Diabetologia 1992: 35: 595.

22. Schanler RJ, Abrams SA, Motil KS, Kim MS. Maternal and economic benefits of breastfeeding, UpToDate 2010; 18: 1.

23. Bhattarai MD. The Prevention of Diabetes: Mother and Child Health. Doha: Diabetes in Asia Study Group, 2012. (Available in the website: http://da-sg.org/download/motherchildhealth.pdf ) 